\title{
Inverse Statistics for Stocks and Markets
}

\author{
A. Johansen* \\ Teglgårdsvej 119, DK-3050 Humlebæk, Denmark \\ I. Simonsen \\ Department of Physics, NTNU, NO-7491 Trondheim, Norway \\ M.H. Jensent \\ Niels Bohr Institute, Blegdamsvej 17, DK-2100 Copenhagen Ø, Denmark
}

(Dated: October 10, 2018)

\begin{abstract}
In recent publications, the authors have considered inverse statistics of the Dow Jones Industrial Averaged (DJIA) 1, 2, 3]. Specifically, we argued that the natural candidate for such statistics is the investment horizons distribution. This is the distribution of waiting times needed to achieve a predefined level of return obtained from detrended historic asset prices. Such a distribution typically goes through a maximum at a time coined the optimal investment horizon, $\tau_{\rho}^{*}$, which defines the most likely waiting time for obtaining a given return $\rho$. By considering equal positive and negative levels of return, we reported in 2, 3] on a quantitative gain/loss asymmetry most pronounced for short horizons. In the present paper, this gain/loss asymmetry is re-visited for $2 / 3$ of the individual stocks presently in the DJIA. We show that this gain/loss asymmetry established for the DJIA surprisingly is not present in the time series of the individual stocks. The most reasonable explanation for this fact is that the gain/loss asymmetry observed in the DJIA as well as in the SP500 and Nasdaq are due to movements in the market as a whole, i.e., cooperative cascade processes (or "synchronization") which disappear in the inverse statistics of the individual stocks.
\end{abstract}

\section{INTRODUCTION}

What drives prices? This question has been studied for centuries with quantitative theories dating back at least to Bachelier [4], who proposed the random walk hypothesis for price trajectories. As prices in general do not become negative, economist later realized that a more realistic framework was obtained by assuming the random walk hypothesis for the logarithm of the price [5]. This has made relative returns the prime focus of financial investigation with only a few exceptions, such as hedge funds focusing on absolute returns, and benchmarking are almost exclusively done by the financial community by comparing relative returns with respect to a fixed time interval.

Within the current economic paradigm - the Efficient Market Hypothesis (EMH) [6] — the idea of randomly fluctuating prices in the absence of new hard information has been re-formulated in the framework of hard-working rational traders with complete knowledge of all available information whose continuing effort more or less instantaneously removes any imbalance in prices due to past differences in the expectations of traders. In short, the EMH states that current prices reflect all available information about the priced commodity, i.e., all available information is at any given instant already priced in by the market and any change in prices can only be due to the revelation of new information. In other words, there is no free lunch.

Hence, the EMH claims that information drives prices. Unfortunately, this just leave us with another question, namely how to price in the available information 21]? In the case of a stock, this must be done by considering how the available information affects future earnings of the company. This obviously (again) introduces some ambiguity as not only do peoples expectations to a largely unpredictable future differ, but so do their strategies with respect to for example investment horizons, i.e. how long they intend to hold their investment before taking any profit, and how large a risk they are willing to take over that time period.

In order to qualify different pricing models etc., the financial industry has performed many statistical studies establishing a number of so-called stylized facts [8, 9, 10] as well as benchmarking for the performance of various financial instruments with respect to investment returns and in its complement, risk taking. Due to this focus on returns and risk, most financial studies essentially amount to measuring two-point correlations in one way or another,

\footnotetext{
*Electronic address: anders-johansen@get2net.dk

${ }^{\dagger}$ Electronic address: Ingve.Simonsen@phys.ntnu.no

‡Electronic address: mhjensen@nbi.dk
} 
most commonly done by studying the distribution of returns calculated over some pre-defined fixed time period $\Delta t[8,9,10]$.

Empirically it has been established, that for not too long time intervals $\Delta t$ 's, say less than a week, the return distributions are characterized by "fat tails" 8, 9, 10]. Fat tails of a distribution refers to a much larger probability for large price changes than what is to be expected from the random walk or Gaussian hypothesis. As $\Delta t$ reaches longer time scales, the distribution of returns gradually converges to a Gaussian distribution. However, no real consensus regarding the exact quantitative nature of this distribution exist.

¿From the point of view of the present authors, a more reasonable answer to the question of what drives prices, besides the influence of interest rates and other macroscopic economic factors as well as quarterly earnings reports etc. of the company in question, is human psychology with all its facets of bounded rationality, limited information, personal beliefs etc. In accordance with this view, the economics and financial communities have in the past 5-10 years witnessed an increased interest in what has been coined "Behaviourial Economics". A prime example of such a study is the book by R.J. Schiller entitled "Irrational Exuberance" 22], published in 2000, but written before the crash of April of this year [11]. This book gives numerous examples of both present (at that time) and past events where the price of stocks, and more relevant, the $\mathrm{P} / \mathrm{E}$ (price-earning-ratio) has more or less exploded based on little more than vague notions of a "new economic era" 23] and the only real difference comes with the specific sector driving the bubble prior to the crashes seen in the past 150 years or so.

If we focus on the US stock market, then in the 1860s and 1870s it was rail road era, in the 1920s it was utilities, semi-conductors ("Tektronix") influenced the development in 1950s and 1960s, in the 1980s it was a general public investment boom driven by liberalization of the financial markets and, most recently, in second half of the 1990s it was first an emerging market boom and then the notorious information technology bubble. Most notable are the "explosions" in the P/E in the 1920s and in the period from early 1980s to 2000. During these periods, the P/E of the SP500 went from about 5 to 32 and from about 7 to 45, respectively [24]. This corresponds to a more than six-fold increase in little less that one and two decades for the two respective periods.

After the October 1987 crash, R.J. Schiller asked a number of institutional and individual investors the following question: "Which of the following better describes your theory of the decline(s): (i) a theory about investor psychology, or (ii) a theory about fundamentals such as profits and interest rates?" The answers revealed that about $68 \%$ of the institutional investors and $64 \%$ of the individual investors picked the former alternative. From this R.J. Schiller concluded that: "It appears that the stock market crash had substantially to do with a psychological feedback loop among the general investing public from price declines to selling and thus further selling, and so forth". This conclusion was in accordance with his investigation of the news stories published immediately prior to the crash of October 1929 where also no specific event or events could be singled out as responsible for the crash.

¿From the point of view of human psychology, the financial communities focus on returns does not make as much sense as one would like. The obvious answer to the question: "What return would you like to see a year from now?", is of course — "As large as possible!" — or, if the question concerned risk — "As small as possible!". A more natural investment strategy is thus to settle for some return level and/or maximum risk and then ask how long one must wait in order to achieve this. But how is one to determine a priori this investment horizon in an utterly unpredictable world with respect to the future some months away? Even if one solely focus on macroscopic fundamentals as well as those of the company in question, the predictability of future earnings is a very difficult question. As J.P. Bouchaud said in a lecture in Granada of February 2005: "I do not know how to price even my own company within a factor of two or perhaps even three though I know everything to be known about it!"

Another, more philosophical problem, with standard models of financial markets is the following: If one, as these models do, only assumes that the market participants are purely selfish individuals, who optimize their own utility function through fixed time contracts with other nominally identical individuals, then, despite the achievement of mutual benefits, the term "contract" would not be defined in a general context. Why is this so?

Because, a general and lasting definition of the term "contract" requires long-term (meaning much longer than an ordinary human time span) institutions, which can only be upheld through non-selfish behavior. Legal institutions, for example, have a life time of centuries and hence cannot be put to work within a framework of selfish individuals who's time horizon can't possibly be longer that there own life span. Hence, most models of the financial markets are lacking a very essential ingredient, which one usually denote by the general word "culture". In conclusion, in our opinion most models of the financial markets lack "psychology and culture".

This does not mean, however, that one cannot ask what drives the economy and the stock market on longer time scales, i.e. over decades, and what behaviour the economy exhibits over these time scales! An example of such a study is presented on Fig. 1] Here, the historical public debt of the USA is shown together with a labeling of the most significant historic events, mostly military conflicts, involving the US during the same period. Notice that the Korean War and it's formal UN-forces did not represent any significant increase in US public debt. However, the so-called Cold War between the USA and the USSR most certainly did. It is clear that the over-all rise in the public debt is exponential driven by large "bumps" signifying rapid increases in public debt. 
It is striking, on a qualitative level, that the origin of these large increases in US public debt is simply related to war with two major exceptions; the purchase of the Louisiana Territory from Napoleon in 1803 on the unauthorized initiative of the US-ambassador in Paris, and the Keynesian (in the meaning massive public investments) "New Deal" of Roosevelt in the 1930s. Note, that the logarithmic scale is the reason why the "bumps" belonging to the purchase of the Louisiana Territory and the Spanish War of 1898 do not "stand out" in Fig. 1

If one compares this figure with one of the US stock market, say, the DJIA (Figs. 2) one clearly sees that on a qualitative level the rises in the public debt, due to wars and New Deal, are followed by steep rises in the stock market with one big exception, namely the bubble of the 1920s. In hind-sight, this may not be so surprising, since increases in public debt normally means large public spending which funnels large amounts into the private sector. However, one should note that the average growth rate of the US public debt is about $8.6 \%$, see Fig. 10 This should be compared with that of the DJIA until the $1950 \mathrm{~s}$, which is about $2.5 \%$. What is surprising is that most time periods with peace exhibit a significant decline in the public debt, most notably the period after the war of 1812 until the second war with the Seminole Indians (1835-1842), as well as modest growth in the DJIA. In the first half of the 19th century, the US public debt dropped to a meager US $\$ 33.733$ in 1835 from US $\$ 75.463 .477$ in 1791 25]. In conclusion with respect to long-term growth in the stock market, public spending, especially in the case of war, has played a very significant role in the long-term growth of the US economy and hence of the DJIA.

In the next sections, we will turn to the subject of optimal investments horizon distributions for both individual stocks as well as markets. We will do this in order to qualify an answer to the previous questions re-formulated as "what a return is reasonable to expect in $x$ days from now" for both individual stocks as well as markets by considering the gain distribution for pre-defined return levels. In order to quantify the risk from historical data, we also consider the corresponding loss distribution 26].

The remaining part of this paper is organized as follows. In the next section, we turn to the short-term behaviour of three US stock markets, namely the DJIA, the SP500 and the Nasdaq. Here, we will re-introduce a conceptually new framework for the analysis of short-term (in the sense of days and weeks) price fluctuations. We call this tool of analysis for inverse statistics as we fix the return level and let time float, and not vice versa, as in the usual return statistics. (It is worth noting that an analysis, where both return level and time floats conditioned on a stable trend in either direction has been published by the first author 13, 14]). First, we re-visit previous results for the DJIA as well as presenting new results for the SP500 and the Nasdaq index, showing that the distribution of time horizons needed to obtain a specified return can be excellently parameterized by a generalized gamma-distribution. Such distributions are well-known from various first-passage problem [15], but the quality of this parametrization is nevertheless surprising considering the nature of the data. We then turn to the previously found gain/loss asymmetry for the DJIA [2] and show that a similar asymmetry is present in both the SP500 and Nasdaq. In the third section, we turn to the use of the inverse statistics on the single stocks that are included in the DJIA in order to further investigate the origin of this gain/loss asymmetry. Surprisingly, we find that the gain/loss asymmetry obtained for the index vanishes for the individual stocks. The last section concludes arguing that the gain/loss asymmetry found in the index comes from a cooperative cascade through the various sectors of the economy represented in the DJIA.

\section{PREVIOUS WORK}

In resent publications 1, 2, 3, 16], the present authors have proposed to invert the standard return-distribution problem and instead study the probability distribution of waiting times needed to reach a fixed level of return $\rho$ for the first time (see also Ref. 17]). As mentioned previously, this is in the literature known as the "first passage time"-problem [15] and the solution is known analytically for a Brownian motion as the Gamma-distribution

$$
p(t)=|a| \frac{\exp \left(-a^{2} / t\right)}{\sqrt{\pi} t^{3 / 2}},
$$

(with $a \propto \rho$ ), where one for large (waiting) times recovers the well-known first return probability for a random walk $p(t) \sim t^{-3 / 2}$.

Historical financial time series such as the DJIA, SP500 and Nasdaq possesses an (often close to exponential, see however [18]) positive drift over long time scales due to the overall growth of the economy modulated with times of recession, wars etc. If such a drift is present in the analyzed time series, one can obviously not compare directly the empirical probability distribution for positive and negative levels of return. As the focus of the present paper will be on such a comparison, we must eliminate or at least significantly reduce the effect of this drift. One possibility for detrending the data is to use so-called deflated asset prices, but such prices are calculated using certain economic assumptions, which we as physicists are naturally suspicious of.

In the present study as well as in those of 1, 2], we have instead chosen to remove the drift based on the use of wavelets [19, 20], which has the advantages of being non-parametric. This technique has been described in detail 
elsewhere 1] and for the present purpose, it suffices to say that this wavelet technique enables a separation of the original time series $s(t)$ into a short scale (detrended) time series $\tilde{s}(t)$ and a (long time-scale) drift term $d(t)$ so that $s(t)=\tilde{s}(t)+d(t)$ (cf. Fig. 2(c)).

Based on $\tilde{s}(t)$ for some historical time period of the DJIA, the empirical investment horizon distributions, $p\left(\tau_{\rho}\right)$, needed to obtain a pre-defined return level $\rho$ for the first time can easily be calculated for different $\rho$ 's. As $\tilde{s}(t)$ is stationary over time scales beyond that of the applied wavelet (for a time larger than say 1000 days) it is straightforward to compare positive and negative levels of return.

As the empirical logarithmic stock price process is known not to be Brownian, we have suggested to use a generalized (shifted) Gamma distribution

$$
p(t)=\frac{\nu}{\Gamma\left(\frac{\alpha}{\nu}\right)} \frac{|\beta|^{2 \alpha}}{\left(t+t_{0}\right)^{\alpha+1}} \exp \left\{-\left(\frac{\beta^{2}}{t+t_{0}}\right)^{\nu}\right\},
$$

where the reasons behind $t_{0}$ are purely technical 27$]$.

The results so far have been very encouraging with respect to excellent parametrization of the empirical probability distributions for three major stock markets, namely DJIA, SP500 and Nasdaq; cf. Figs. 3(a), 4(a) and 51 for examples using a return level of $5 \%$ and the figure captions for values of the fit parameters. The choice of $\rho= \pm 0.05$ is not accidental. It is sufficiently large to be above the "noise level" quantified by the historical volatility and sufficiently small to be of quite frequently occurrence. We have also considered other return levels, showing qualitatively the same features, but there results are not shown.

In all three cases, the tail-exponents $\alpha+1$ of the distributions parameterized by Eq. (2) are indistinguishable from the "random walk value" of $3 / 2$, which is not very surprising. What is both surprising and very interesting is that these three major US stock markets (DJIA, SP500 and Nasdaq) exhibit a very distinct gain/loss asymmetry, i.e., the distributions are not invariant to a change of sign in the return $\rho$ (Figs. 31(a), 4(a) and 5). Furthermore, this gain/loss asymmetry quantified by the optimal investment horizon defined as the peak position of the distributions has for at least the DJIA a surprisingly simple asymptotically power law like relationship with the return level $\rho$, see Fig. 6

See Ref. 16] for an application of inverse statistics to high-frequency foreign exchange data, specifically the US\$ against the Deutch Mark and the Japanese Yen. We are also currently investigating the use of inverse statistics for intra-day stock prices, but the results so far are still preliminary.

\section{NEW RESULTS}

As mentioned previously, the purpose of the present paper is to further investigate the origin of the gain/loss asymmetry in DJIA. We do that by simply comparing the gain and loss distributions of the DJIA with the corresponding distributions for a single stocks in the DJIA as well as their average.

An obvious problem with this approach is that the stocks in the DJIA changes with historical period and hence an exact correspondence between the DJIA and the single stocks in the DJIA is difficult to obtain if one at the same time wants good statistics. This is the trade-off, where we have put the emphasis on good statistics. However, if this choice produces interpretational difficulties, then one must ask why analyze the historical DJIA at all?

The 21 company stocks analyzed and presently in the DJIA (by the change of April 2004) are listed in table 1 together with the their date of entry into the DJIA as the time period of the data set analyzed.

As previously mentioned, the empirical distributions for $\rho= \pm 0.05$ are presented in Figs. 31(a), 41(a) and 5] for the DJIA, the SP500 and the Nasdaq respectively for the entire time span available to the authors. Furthermore, in Figs. 3(b) and 4(b) we have truncated the full data sets of the DJIA and SP500 to a shorter historical time period in order to compare the results for the indices with that of the individual stocks. What one should primarily note in the comparison between the two sets of figures, i.e., the longer data sets with the shorter, is that the only significant difference is to be found in the weaker statistics of the shorter periods; the positions of the maxima for the positive and the negative gains are in both cases roughly just below 10 and 20 days, respectively, i.e., a difference of roughly a factor of 2 for all three indices for a return level of $5 \%$.

In Figs. [7 we show the waiting time distributions for 4 companies in the DJIA, which are representative for the distributions obtained for all the companies listed in Table W We see that, for a return level $|\rho|=0.05$, the value of the optimal investment horizon, i.e. the position of the peak in the distribution, ranges from around 2 days to around 10 days depending on the company. More importantly, it is clear from just looking at the figures that, within the statistical precision of the data, the distributions are the same for positive and negative values of $\rho$.

In order to further quantify this invariance with respect to the sign of $\rho$, we have averaged the (company) gain and loss distributions separately in order to obtain an average behavior for the stocks listed in Table The result of this averaging process (Fig. 8) is nothing less that an almost perfect agreement between the gain and loss distributions with 
a peak position around 5 days for both distributions. This means that the optimal investment horizon for the average of these selected individual stocks is approximately half that of the loss distribution for the DJIA and approximately one fourth of that for the gain distribution. In other words, it is twice as slow to move the DJIA down and four times as slow to move the DJIA up compared to the average time to move an individual stock in the DJIA up or down.

How can we rationalize these results? What we have done in essence is to interchange the operations of averaging over the stocks in the DJIA and calculating the inverse statistics for the stocks of this index. Since the DJIA is constructed such that it covers all sectors of the economy of which it seems quite reasonable to assume that a $5 \%$ gain/loss in the shares of for example Boeing Airways in general has nothing fundamentally to do with a corresponding gain/loss in the shares of Coca-Cola Company especially since the data are detrended. In other words, it seems quite reasonable to assume that there is nothing special about a return level of $5 \%$ in terms of economic fundamentals etc. This assumption is also strongly supported by the results presented in Fig. [6]

This then means that the two operations, i.e. the averaging and the inverse statistics calculation, do not commute not even approximately. Hence significant inter-stock correlations must exist even for a rather modest return level $\rho=0.05$. In our view, this is a quite surprising result and especially considering that the large differences in the optimal investment horizons for the distributions of the index and the average of the individual stocks.

\section{SUMMARY AND CONCLUSIONS}

We have considered inverse statistics for financial data. It is argued that the natural candidate for such statistics is what we call the investment horizon distribution. Such a distribution, obtained from the historic data of a given market, indicates the time span an investor historically has to wait in order to obtain a predefined level of return. For the three major US markets, namely the DJIA, SP500 and Nasdaq, the distributions are parametrized excellently by a shifted generalized Gamma distributions for which the first moment does not exist.

The typical waiting time, for a given level of return $\rho$, can therefore be characterized by e.g. the time position of the maximum of the distribution which we call the optimal investment horizon. By studying the behaviour of this quantity for positive (gain) and negative (loss) levels of return, a very interesting and pronounced gain/loss asymmetry emerges for all three markets considered. The nature of this gain/loss asymmetry was further investigated by calculating the investment horizon distribution for 21 of the 30 individual stocks in the DJIA. Quite surprisingly the observed gain/loss asymmetry in the DJIA is not present in the investment horizon distribution for the individual stocks.

Specifically, we have shown that if one first "average" the stock prices of, say, the DJIA in order to create the index and then calculate the inverse statistics of it, then one obtains a pronounced gain/loss asymmetry. Reversing the order of these two operations, however, makes this asymmetry disappear. Admittedly, this has been done systematically for only a single gain and loss level $\rho$, but it seems unreasonable to claim anything special about the $5 \%$ gain/loss level used. Hence, the investment horizon distribution for the individual stocks is invariant under a change of sign for $\rho$.

Furthermore, the optimal investment horizon for the average of the distributions for the individual stocks is approximately half that of the loss distribution for the entire market (DJIA) and approximately one fourth of that for the gain distribution for the entire market. In other words, it is twice as slow to move the DJIA down and four times as slow to move the DJIA up compared to the average time to move an individual stock in the DJIA up or down.

There are several possible scenarios which may explain the observed behavior. However, they all amount to more or less the same thing. A down/up-turn in the DJIA may be initiated by a down/up-turn in some particular stock in some particular economical sector. This is followed by a down/up-turn in economically related stocks in other sectors and so forth. The result is a cascade, or synchronization, of consecutive down/up-turns in all the sectors covered by the DJIA. The initiation of this may be some more general new piece of information, which is considered more crucial for one sector than other sectors, but as argued for in length in [11] it may also happen for no obvious reason what so ever.

An (rational) example would be that Intel drops significantly due to bad quarterly earnings in turn, by a cascade process, affecting the stock price of IBM and MicroSoft and so forth. As the index, at least from a physicist's point of view, can be compared to an external field, movements in the index due to a single or a few stocks can rapidly spread through most or all sectors, if psychology in general and specifically feed-back loops are important, thus creating a relatively large movements in the over-all index. That losses in general are faster than gains must also be attributed to human psychology: people are in general much more risk adverse than risk taking [28].

In conclusion, the results presented here thus provide further evidence for the presence of cooperative behavior (or synchronization) possibly with a psychological origin in the stock market beyond what can be deduced from standard financial models. 


\section{Acknowledgment}

I.S. would like to thank the Nordic Institute for Theoretical Physics (NORDITA) for kind hospitality where the last part of this work was completed.

[1] I. Simonsen, M. H. Jensen and A. Johansen, Eur. Phys. J. Vol. 27 No. 4, p. 583 (2002)

[2] M. H. Jensen, A. Johansen and I. Simonsen, Physica A 234 pp. 338-343 (2003)

[3] I. Simonsen, A. Johansen, and M.H. Jensen. Investment horizons : A time-dependent measure of asset performance. In H. Takayasu, editor, Practical Fruits of Econophysics. Springer Verlag, 2005. To appear.

[4] L. Bachelier, Théorie de la Spéculation", 1900, Annales de l'Ecole normale superiure.

[5] P. Samuelson, Economics: The Original 1948 Edition, McGraw-Hill/Irwin; 1 edition (December 1, 1997)

[6] E. Fama, J. Finance, 25, p. 383 (1970). E. Fama, J. Finance, 46, p. 1575 (1991).

[7] A. Johansen, "Discrete scale invariance and other cooperative phenomena in spatially extended systems with threshold dynamics", Ph.D. thesis Niels Bohr Inst. 1998.

[8] J.-P. Bouchaud and M. Potters, Theory of Financial Risks: From Statistical Physics to Risk Management (Cambridge University Press, Cambridge, 2000).

[9] R. N. Mantegna and H. E. Stanley, An Introduction to Econophysics: Correlations and Complexity in Finance (Cambridge University Press, Cambridge, 2000).

[10] J. Hull, Options, Futures, and other Derivatives, 4th ed. (Prentice-Hall, London, 2000).

[11] R. J. Schiller, Irrational Exuberance, Princeton University Press, 2000

[12] A. Johansen and D. Sornette, Eur. Phys J. B 17 pp. 319-328 (2000).

[13] A. Johansen and D. Sornette Eur. Phys. J. B 1, pp. 141-143 (1998)

[14] A. Johansen and D. Sornette, Journal of Risk, 4(2), 69-110 (2001/2002).

[15] S. Redner, A Guide to First Passage Processes (Cambridge, New York, 2001).

[16] M. H. Jensen, A. Johansen, F. Petroni and I. Simonsen, Physica A 340, 678 (2004).

[17] M.H. Jensen, Phys. Rev. Lett. 83, 76-79 (1999).

[18] A. Johansen and D. Sornette, Physica A 294, Issue 3-4, pp. 465-502 (2001).

[19] W.H. Press, S.A. Teukolsky, W.T. Vetterling, and B.P. Flannery, Numerical Recipes in Fortran, 2nd ed. (Cambridge University Press, New York, 1992).

[20] I. Simonsen, A. Hansen, and O. Nes, Phys. Rev. E 58, 2779 (1998).

[21] As the efficient market hypothesis only speaks about the relation between prices and available information it as a consequence can only be tested jointly with some asset-pricing model. This introduces a ambiguity in how to interpret anomalous behaviour of returns: Is the market inefficient to some extent or do we have a bad pricing model? This is in fact a very serious problem, since it makes it very difficult or impossible to "prove" that the market is efficient or inefficient and to some extent turns the entire question into a matter of belief [7].

[22] The title is coined from the label that A. Greenspan put on the development of the stock markets in a speech on December $5,1996$.

[23] Even the terminology characterizing the economy prior to for example the bubbles preceding the crashes of 1929 and 2000 was more or less the same [12].

[24] The average $\mathrm{P} / \mathrm{E}$ is today around 16 for US stocks, which is still the highest in western markets.

[25] In 1790, the Federal Government declared that it was redeeming the Scrip Money that was issued during the Revolutionary War in the amount of US\$ 80.000.000.

[26] This can in fact also be turned into a gain distribution by "shorting".

[27] Its actual value depends on possible short-scale drift which may in part be due to the fact that we are using the daily close. Ideally, one should use some measure of all prices during the day, but it's not obvious how to define such a representative price for the entire trading day. We are aware that the mid price often is used as proxy but it is not obvious that this measure is to be preferred as relatively large price changes tend to occur in the beginning and end of the trading day.

[28] The reason for this has an evolutionary origin: one of the functions of emotions is to speed up decisions, which is an excellent feature in terms of physical survival. However, in the case of the stock markets, where financial survival may be at stake, rational decisions are to be prefered. But, as rational decisions take longer time to make, people will often act on emotions. In a study, published in June in the journal Psychological Science by a team of researchers from Stanford University, Carnegie Mellon University, and the University of Iowa, a group of functional psychopats outperformed a group of people with normal emotional responses in a financial game. 


\begin{tabular}{|l||ll|}
\hline Company & Entering date & Data period \\
\hline \hline Alcoa $^{\star}$ & Apr 22, 1959 & $1962.1-1999.8$ \\
ATTerican Express Company $^{\dagger}$ & Aug 30, 1982 & $1977.2-1999.8$ \\
Boeing Airways $^{\text {Citicorp }}$ & Mar 14, 1939 & $1984.1-1999.8$ \\
Coca-Cola Company $_{\text {DuPont }}$ & Jul 08, 1986 & $1962.1-1999.8$ \\
Exxon \& Mobil & Mar 17, 1997 & $1977.0-1999.8$ \\
General Electric & Mar 12, 1987 & $1970.0-1999.8$ \\
General Motors & Nov 20,1935 & $1962.1-1999.8$ \\
Goodyear & Oct 01, 1928 & $1970.0-1999.8$ \\
Hewlett \& Packard & Nov 07, 1907 & $1970.0-1999.8$ \\
IBM & Mar 16, 1915 & $1970.0-1999.8$ \\
Intel & July 18 1930 & $1970.1-1999.8$ \\
International Paper & Mar 17, 1997 & $1977.0-1999.8$ \\
Eastman Kodak Company & Jun 29, 1979 & $1962.0-1999.8$ \\
McDonald's Cooperation & Nov 01, 1999 & $1986.5-1999.8$ \\
Merck \& Company & Jul 03, 1956 & $1970.1-1999.8$ \\
Procter \& Gamble & Oct 30, 1930 & $1962.0-1999.8$ \\
The Walt Disney Co. & Jun 29, 1979 & $1970.1-1999.8$ \\
Wall Mart & May 26, 1932 & $1970.0-1999.8$ \\
\hline
\end{tabular}

TABLE I: List of the (21) DJIA stocks analyzed in this work (about $70 \%$ of the total number). Furthermore, their date of entry into the DJIA are shown, and the time period covered by the analyzed data set. All of these companies are also naturally part of SP500 with General Electric as the most heavily weighted stock. ${ }^{\star}$ Former Aluminum Corporation of America. ${ }^{\dagger}$ Former American Tel. \& Tel. Truncated due to anti-trust case in 1984. ${ }^{\bullet}$ Former Travelers Group. ${ }^{\circ}$ Former Standard Oil.

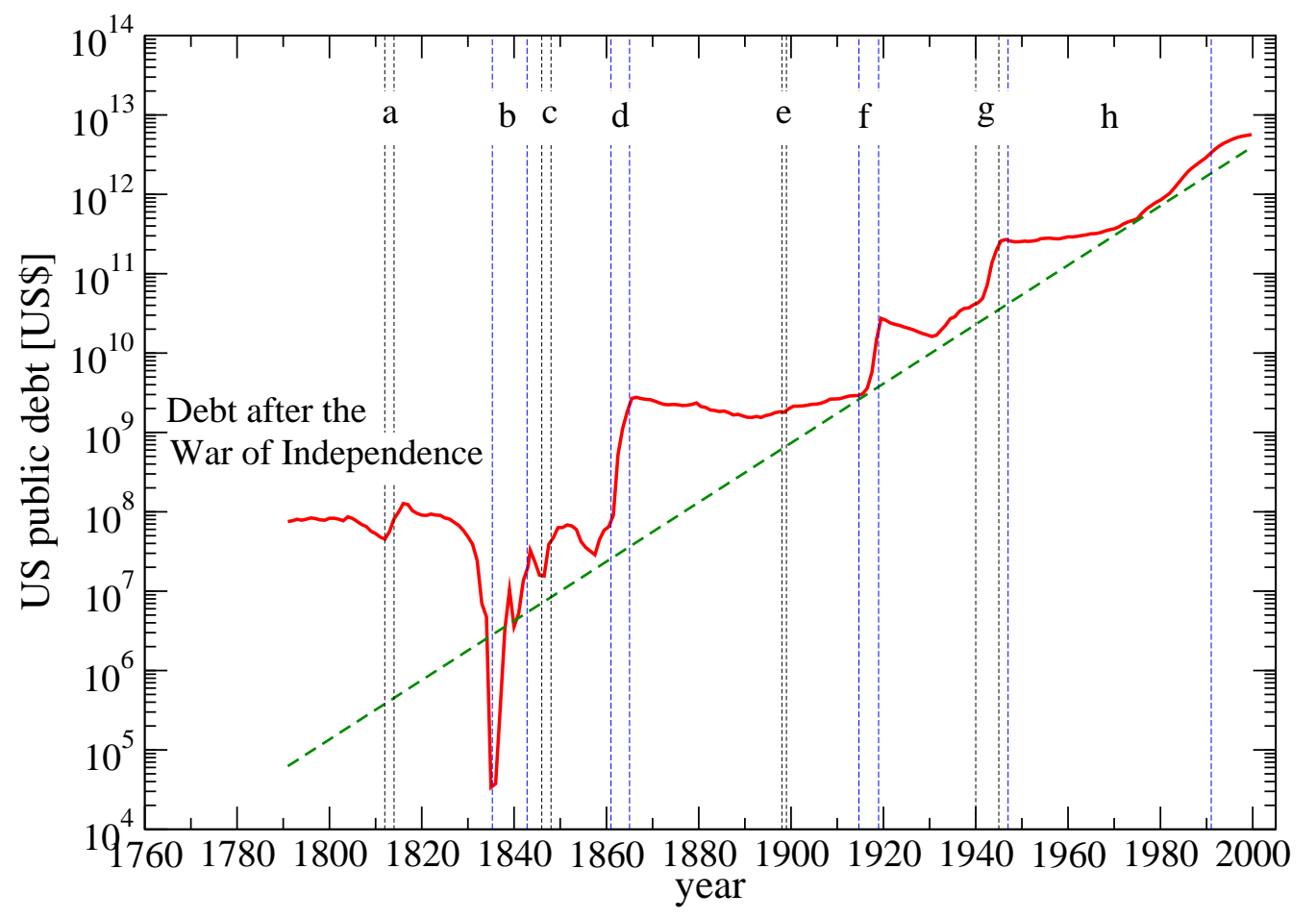

FIG. 1: Graph of the historical US public debt from 1791 till 2000. The dashed diagonal line represents an exponential function corresponding to an average growth rate of about $8.6 \%$. Some historic events are marked by dashed vertical lines in the figure. They are: (a) the 1812 war (1812-1814); (b) the second war with the Seminole Indians (1835-1842); (c) The Mexican-American War (1846-1848); (d) The Civil War (1861-1865); (e) The Spanish American War (1898); (f) The First World War (1914-1918); (g) The Second World War (1940-1945) (h) The Cold War (1947-1991). 


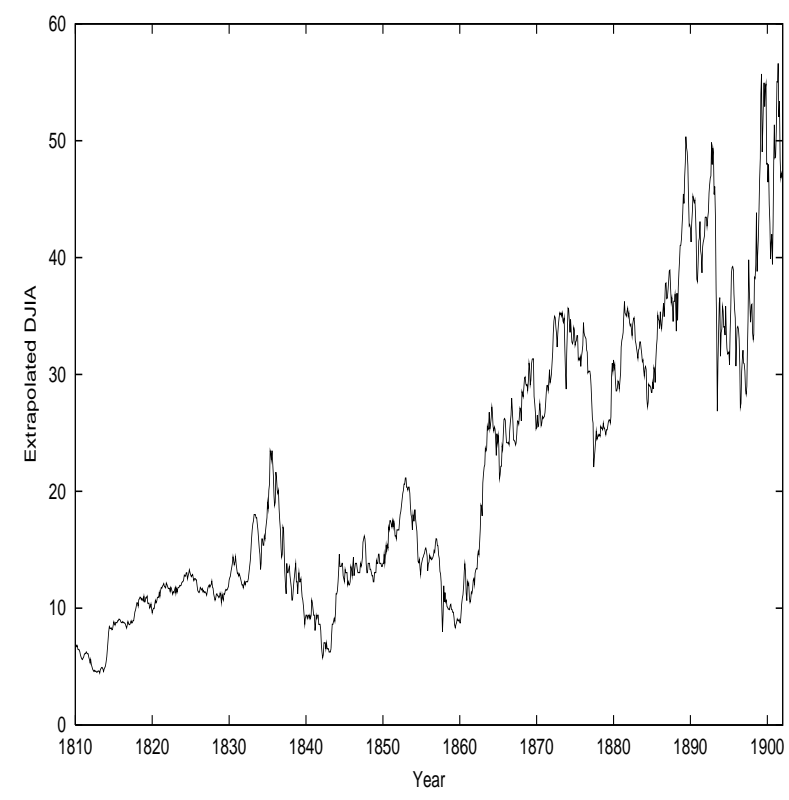

(a) DJIA 1810-1902

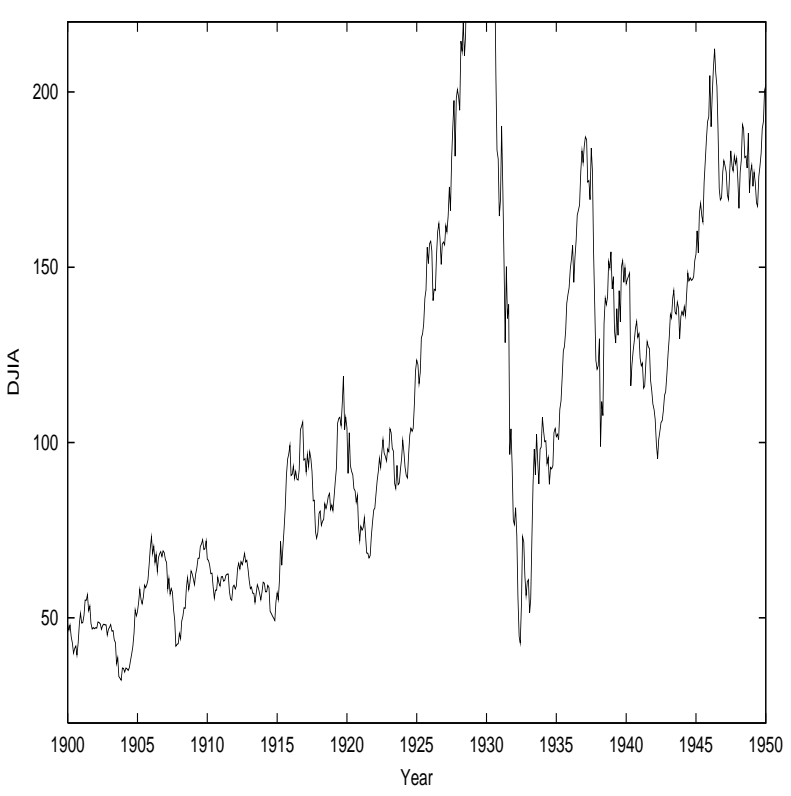

(b) DJIA 1900-1950

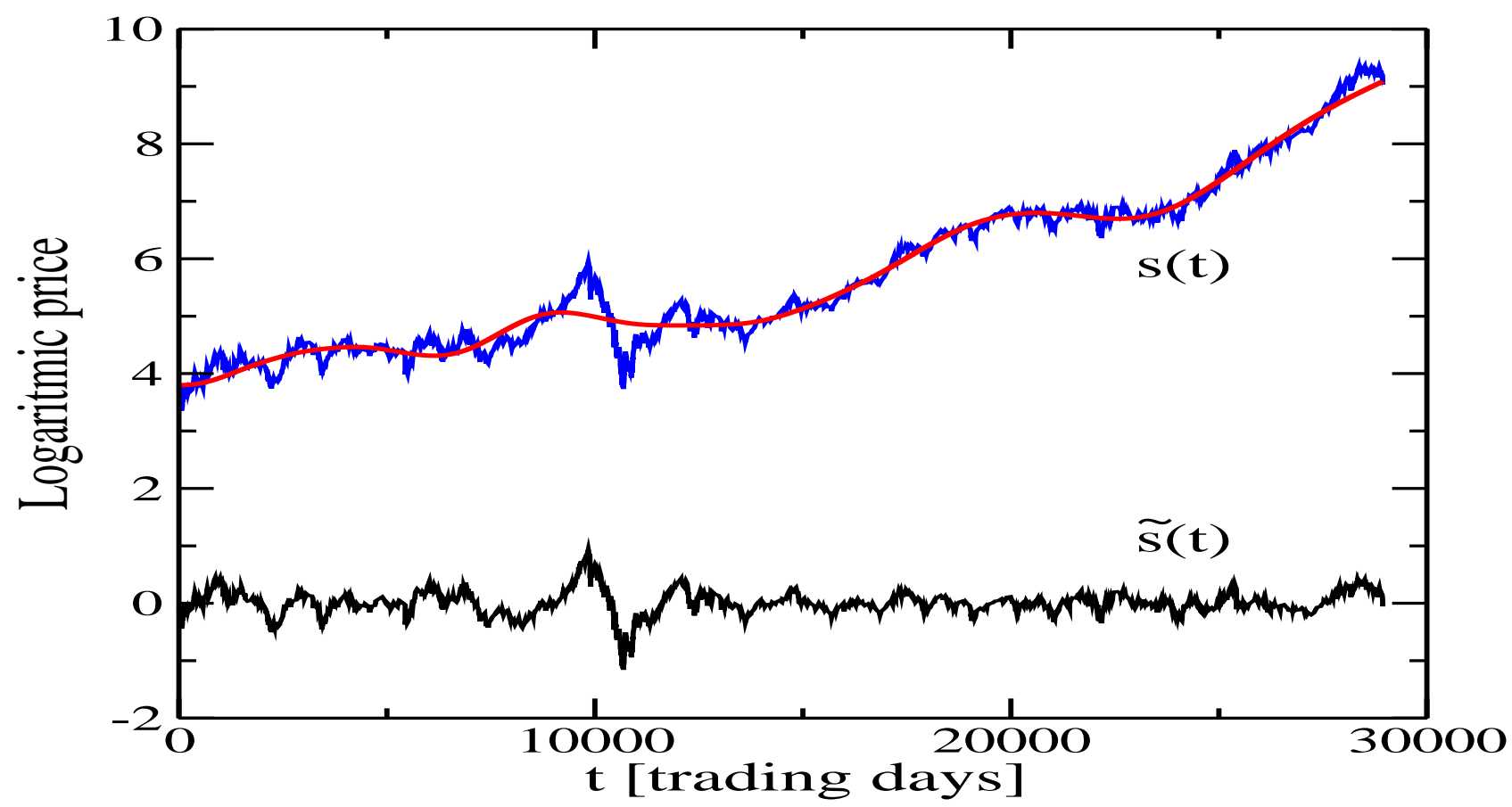

(c) DJIA 1896-2001 (incl. detrended data)

FIG. 2: Historic DJIA data: (a) 1810-1902; (b) 1900-1950; (c) 1896-2001 including the (wavelet) detrended data series, $\tilde{s}(t)$, analyzed below (cf. Ref. 1] for further details). 


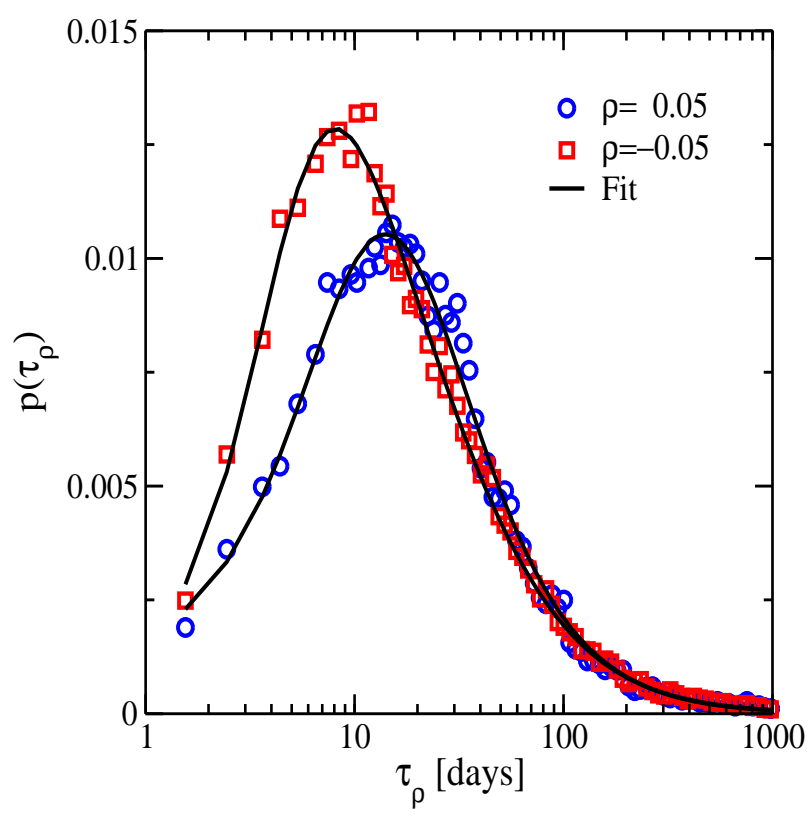

(a) DJIA (1896.5-2001.7)

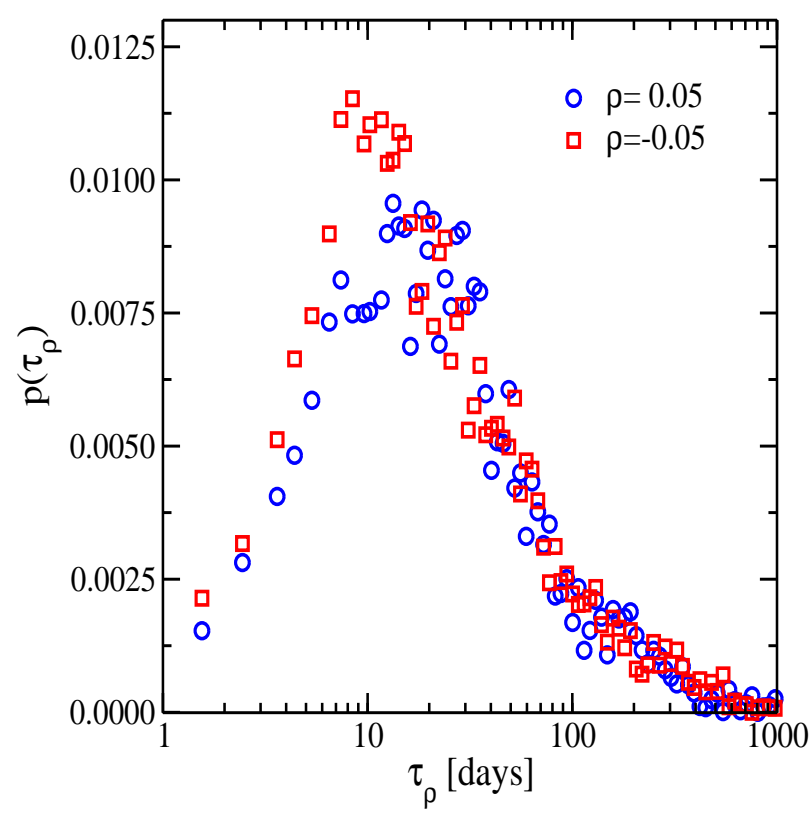

(b) DJIA (1960.8-1999.8)

FIG. 3: Inverse statistics for detrended closing prices (open symbols) of the DJIA for the time periods indicated. For all cases the return levels used were $|\rho|=0.05$. The solid lines represent the best fits of Eq. ([2) to the empirical data with the parameters indicated below; (a) DJIA (1896.5-2001.7): $\alpha \approx 0.51, \beta \approx 5.23, \nu \approx 0.68$ and $t_{0} \approx 0.42$ (loss distribution); $\alpha \approx 0.51$, $\beta \approx 4.53, \nu \approx 2.13$ and $t_{0} \approx 10.1$ (gain distribution); (b) Same as Fig. 31a), but for a shorter time period (1960.8-1999.8). Note that the tail exponents $\alpha+1$ are very close to the "random walk value" of $3 / 2$ for all distributions. 


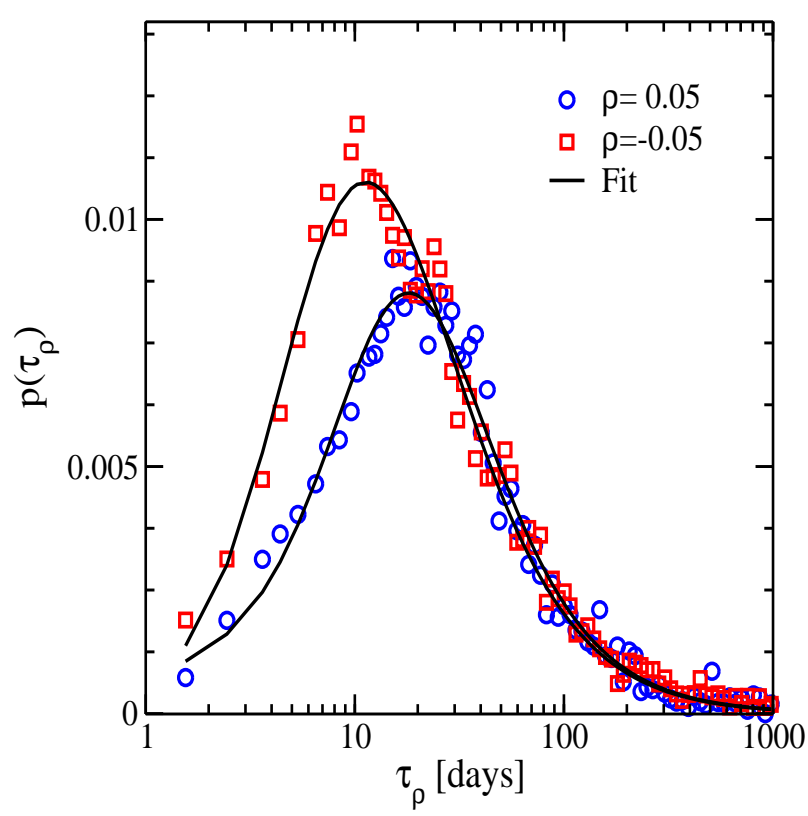

(a) SP500 (1940.9-2000.3)

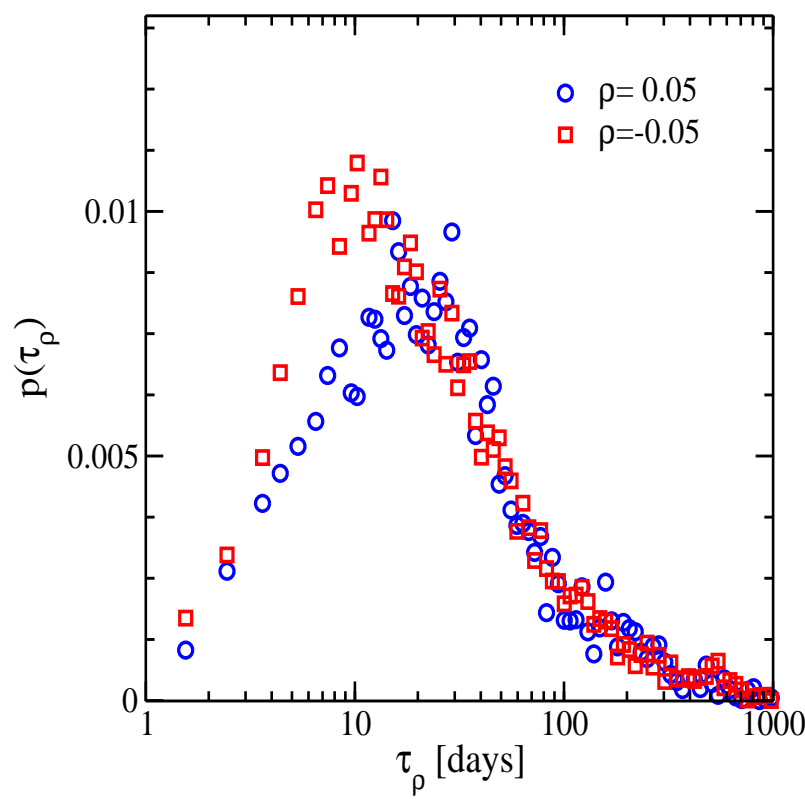

(b) SP500 (1960.8-2000.3)

FIG. 4: Inverse statistics for detrended closing prices (open symbols) of the SP500 for the time periods indicated. For all cases the return levels used were $|\rho|=0.05$. The solid lines represent the best fits of Eq. (2) to the empirical data with the following parameters: (a) SP500 1940.9-2000.3): $\alpha \approx 0.50, \beta \approx 4.87, \nu \approx 0.88$ and $t_{0} \approx 1.59$ (loss distribution); $\alpha \approx 0.50, \beta \approx 5.10$, $\nu \approx 2.56$ and $t_{0} \approx 14.0$ (gain distribution); (b) Same as Fig. 4l(a), but for a shorter time period (1960.8-1999.8). Note that the tail exponents $\alpha+1$ are very close to the "random walk value" of $3 / 2$ for all distributions. 


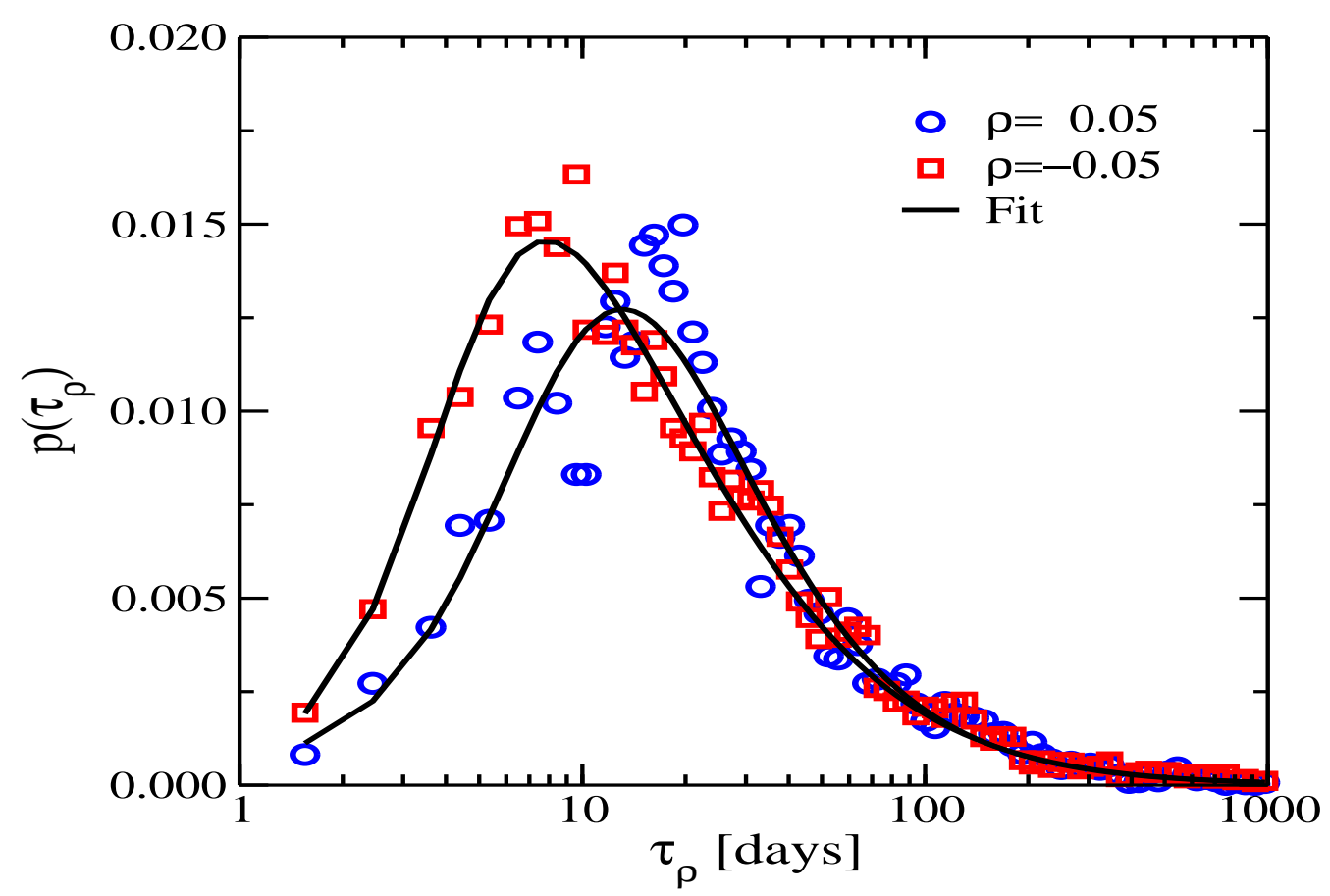

FIG. 5: Same as Fig. 3(a), but for the Nasdaq. The historical time period considered is 1971.2 to 2004.6. Again he solid lines represent fit of the empirical data against Eq. (2) with parameters: $\alpha \approx 0.51, \beta \approx 4.72, \nu \approx 0.73$ and $t_{0} \approx 7.92$ (loss distribution); $\alpha \approx 0.51, \beta \approx 4.16, \nu \approx 2.41$ and $t_{0} \approx 0.07$ (gain distribution). Note again that the tail exponents $\alpha+1$ are very close to the "random walk value" of $3 / 2$ for both distributions.

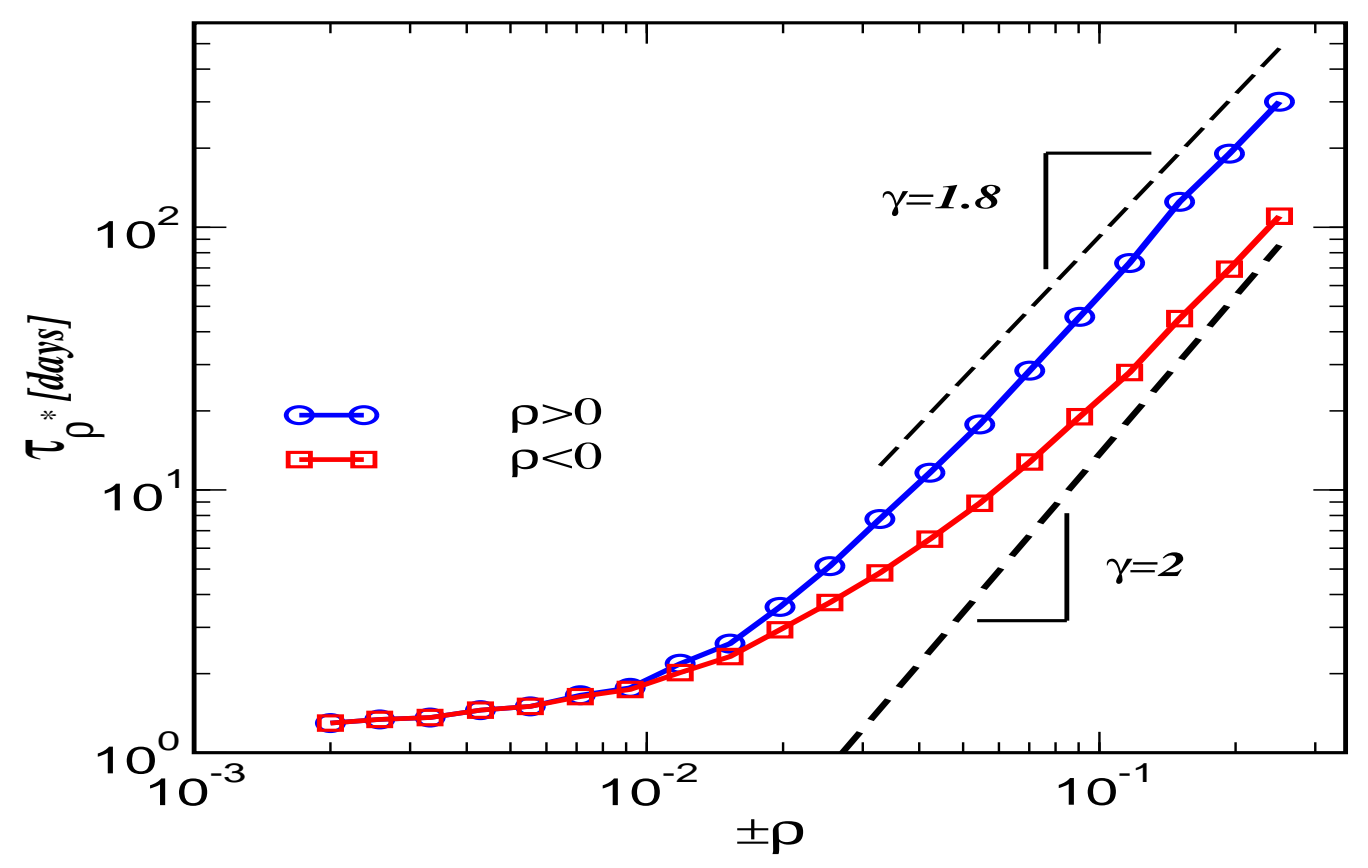

FIG. 6: The optimal investment horizon $\tau_{\rho}^{*}$ for positive (open circles) and negative (open squares) levels of return $\pm \rho$ for the DJIA. In the case where $\rho<0$ one has used $-\rho$ on the abscissa for reasons of comparison. If a geometrical Brownian price process is assumed, one will have $\tau_{\rho}^{*} \sim \rho^{\gamma}$ with $\gamma=2$ for all values of $\rho$. Such a scaling behaviour is indicated by the lower dashed line in the graph. Empirically one finds $\gamma \simeq 1.8$ (upper dashed line), only for large values of the return. 


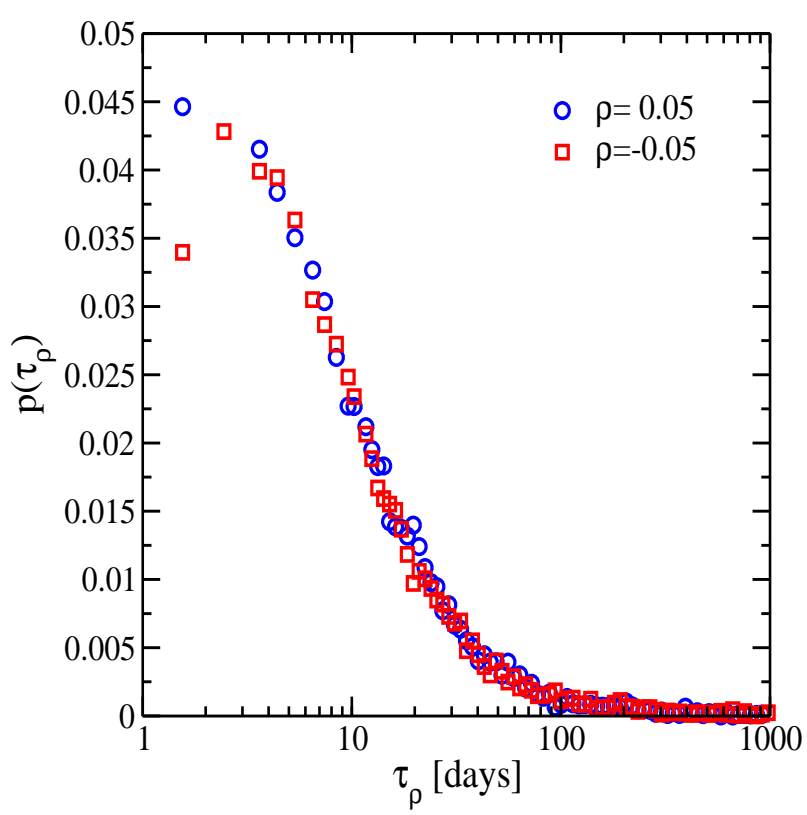

(a) Boeing Airways

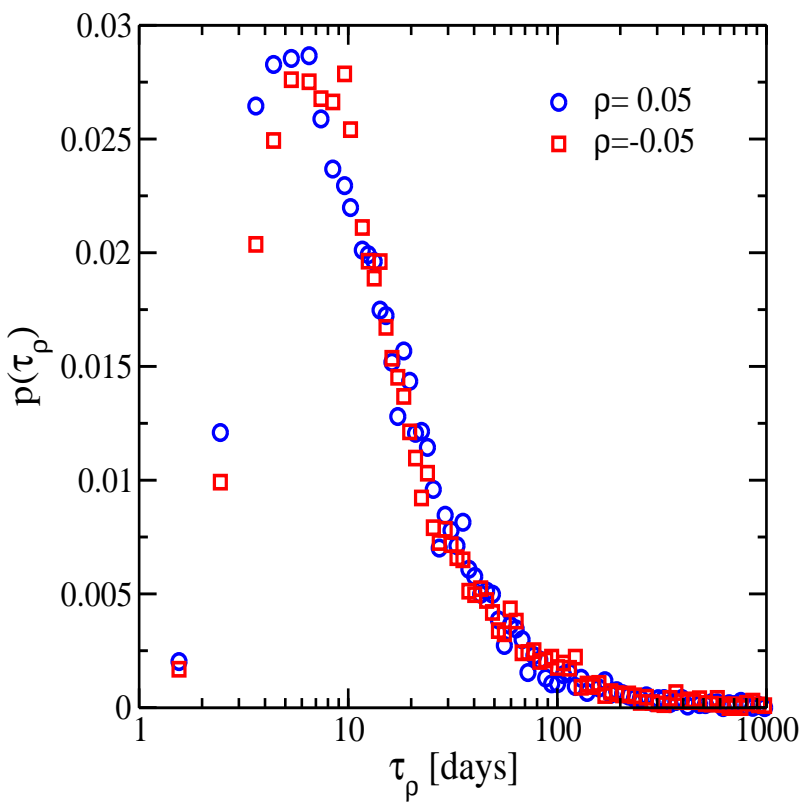

(c) General Motors

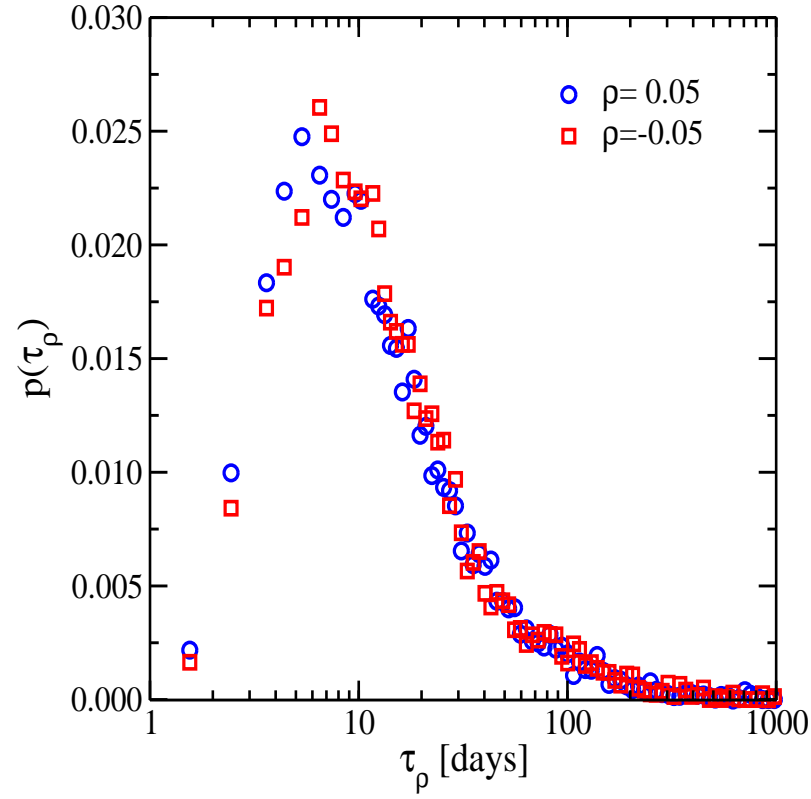

(b) General Electric

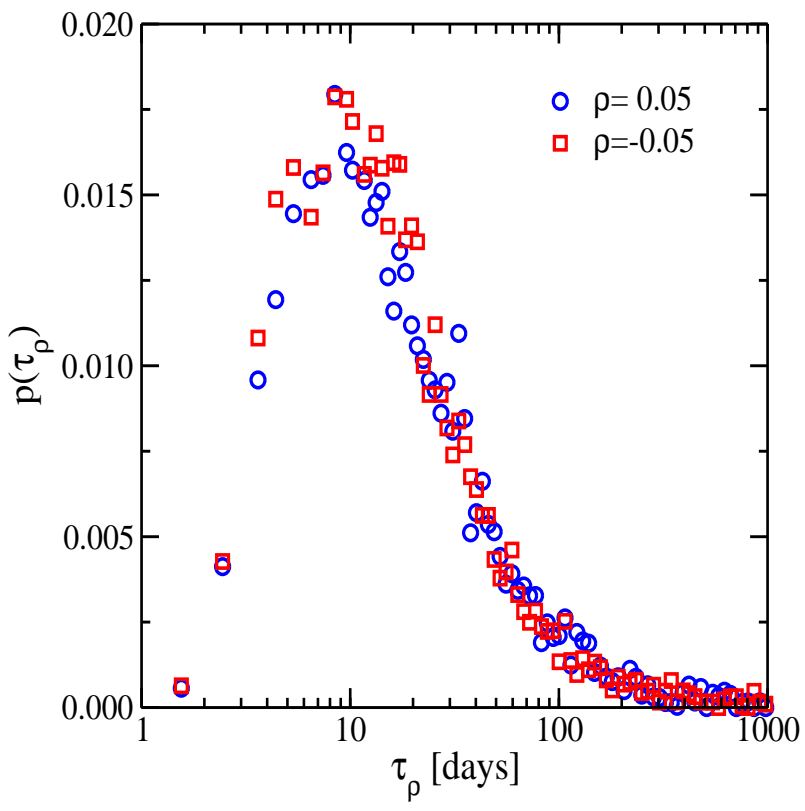

(d) Exxon \& Mobil

FIG. 7: Same as Fig. 3(a), but for some of the individual companies of the DJIA: (a) Boeing Airways (1962.1-1999.8); (b) General Electric (1970.0-1999.8); (c) General Motors (1970.0-1999.8); (d) Exxon \& Mobil, former Standard Oil (1970.0-1999.8). 


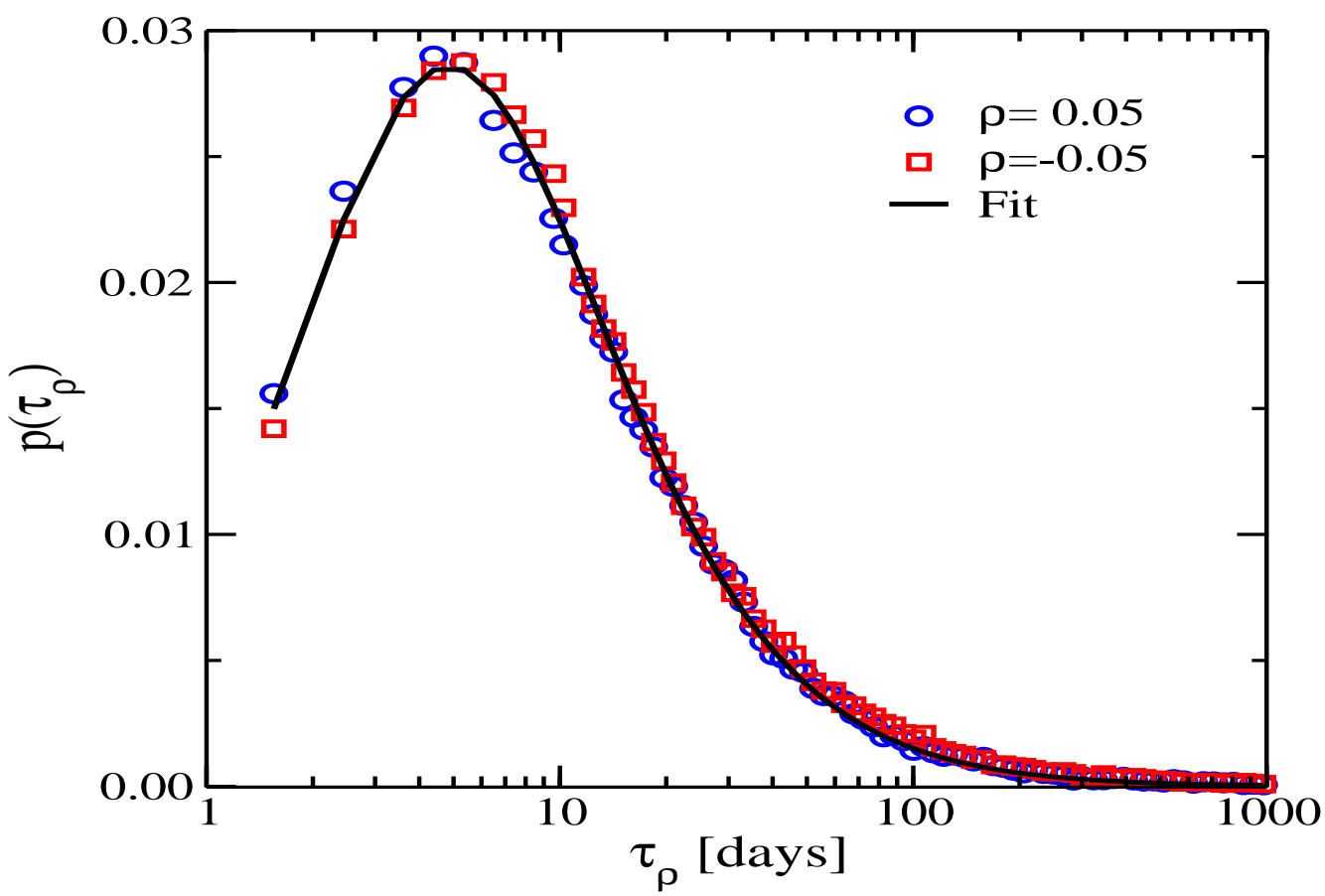

FIG. 8: Averaged gain and loss distribution for the companies listed in table \ The fit is Eq. (2) with values $\alpha \approx 0.60$, $\beta \approx 3.24, \nu \approx 0.94$ and $t_{0} \approx 1.09$. Note that the tail exponent $\alpha+1$ is 0.1 above the "random walk value" of $3 / 2$. 